\title{
Multiferroics: Looking back and going forward
}

\author{
LIU YunYa ${ }^{1} \&$ LI JiangYu ${ }^{2 *}$ \\ ${ }^{1}$ Key Laboratory of Low Dimensional Materials and Application Technology of Ministry of Education, School of Materials Science and \\ Engineering, Xiangtan University, Xiangtan 411105, China; \\ ${ }^{2}$ Department of Materials Science and Engineering, Southern University of Science and Technology, Shenzhen 518055, China
}

Received September 26, 2020; accepted November 2, 2020; published online November 10, 2020

Citation: Liu Y Y, Li J Y. Multiferroics: Looking back and going forward. Sci China Tech Sci, 2020, 63: 2735-2736, https://doi.org/10.1007/s11431-020-1742-7

Multiferroics refer to a material system with the coexistence of two or more ferroic orders, such as ferroelastic order, ferroelectric order, and magnetic order [1]. The couplings among different ferroic orders result in the multi-field couplings among mechanical, electric, and magnetic fields, allowing multiferroics to exhibit fascinating multi-functional properties [2]. In particular, the attractive magnetoelectric coupling makes it possible to manipulate polarization by an external magnetic field, or to control magnetization by an external electric field, offering an intriguing mechanism for devices, such as data storage, magnetoelectric sensors, and other potential technological products.

Magnetoelectric coupling does not follow time-reversal symmetry, and thus in the earlier days, it was postulated that magnetoelectric coupling did not exist in materials, until Landau and Lifshitz found that time reversal is not a symmetry operation in some magnetic crystals [3], inferring that magnetoelectric coupling may exist in spin ordered crystals. The existence of magnetoelectric coupling was first predicted in chromium oxide by Dzyaloshinskii [4]. Then magnetoelectric coupling, in the form of induction of magnetization by an electric field, was first detected by Astrov [5] in the experiments. Subsequently, the magnetoelectric coupling, in the form of induction of polarization by a magnetic field, was observed in chromium oxide in the experiments by Rado and Folen [6]. For a long time afterward, some single-phase multiferroics have also been discovered,

*Corresponding author (email: lijy@sustech.edu.cn) and the requirements of structure and symmetry for a crystal to possess multiferroicity were summarized by Schmid [1]. However, most of the single-phase multiferroics show very week magnetoelectric coupling only at low temperature. To achieve magnetoelectric coupling near room temperature, multiferroic composites, composed of a ferroelectric constituent and a magnetic constituent, have been designed for generating magnetoelectric coupling through the product effect between piezoelectric and piezomagnetic (magnetostrictive) effects, which are connected by the elastic field in composites [7]. The room-temperature magnetoelectric coupling discovered in bismuth ferrite renewably has ignited interests to the studies of single-phase multiferroics [8]. In 2005, Fiebig [9] reviewed the revival of magnetoelectric effect from the research activities on the linear magnetoelectric effect. Some of the progressive milestones in singlephase multiferroics were outlined by Wang et al. [10], and various strategies for combining magnetic and polarization orders were also summarized. With new theories, advanced computational methods, novel material synthesis and processing, and precise characterization techniques, multiferroics are experiencing rapid development with the discoveries of new structures, new physical phenomena, and new mechanisms, which foster potentially novel technological applications of multiferroics [11].

Recently, the recent development, current challenges, and opportunities in multiferroic materials have been reviewed. The rapid development of technologies in material growth has emerged various new multiferroic material structures 
[11]. For multiferroic composites, Hu et al. [12] described the mechanisms for realizing the voltage control of magnetization in four types of multiferroic heterostructures, remarking also the corresponding current trends and some future directions. Other than multiferroic composites, singlephase multiferroics appear to be advantageous in miniaturizing magnetoelectric devices, which attracts the discoveries of novel single-phase multiferroic materials. Lu et al. [13] reviewed the recent progress on single-phase multiferroic materials in the aspects of new materials, phenomena, and physics, as well as the efficient pathways for the enhancement of performance.

Multiferroic materials show various physical performances, especially magnetoelectric properties, underpinned by their microscopic domain structures, such as ferroelastic, ferroelectric, and magnetic domains, and their domains interaction and switching under external field stimuli. Scanning probe microscopy (SPM) has emerged as one of the most powerful tools for characterizing and manipulating domain structures and multi-field couplings of multiferroics at the nanoscale. Liu et al. [2] focused on several SPM techniques, such as piezoresponse force microscopy (PFM), magnetic force microscopy (MFM), magnetoelectric force microscopy (MeFM), and conductive atomic force microscopy (c-AFM), and also highlighted the challenges, opportunities, and outlook of multiferroic materials probed by a SPM tip.

Low dimensional multiferroics are beneficial for the miniaturization of magnetoelectric devices, and also exhibit novel domain structures, especially topological domains and conductive domain walls, which are promising in the implementations of fast high-density and nonvolatile data storage devices. Tian et al. [14] reviewed the recent advances in topological domain states in size-confined multiferroic nanostructures, and also summarized the electric-fielddriven magnetic switching through domain engineering. The charge accumulation and the changes in the local band gap usually occur in topological domain states. Structures and electronic properties of domain walls in $\mathrm{BiFeO}_{3}$ thin films, as one of the most widely studied single-phase multiferroic, have been reviewed by Huyan et al. [15], and the correlation among conductivity, photovoltaic properties, atomic-scale structures, and dynamic of domain walls were also men- tioned, showing great potential of the applications in the next-generation nanodevices.

Fascinating developments of new material systems, new physical phenomena, and new physical mechanism have appeared in multiferroic materials. Devices based on multiferroic materials have also been proposed. Nevertheless, practical applications of multiferroics-based devices remain challenging but offer a driving force for the research on newtype multiferroic materials, enhancement of multiferroic performance, and development of related devices.

This work was supported by the National Natural Science Foundation of China (Grant Nos. 11772286 and 11627801), Huxiang Young Talents Plan Project of Hunan Province (Grant No. 2019RS2037), and the Leading Talents Program of Guangdong Province (Grant No. 2016LJ06C372).

1 Schmid H. Multi-ferroic magnetoelectrics. Ferroelectrics, 1994, 162: 317-338

2 Liu Y, Seidel J, Li J. Multiferroics under the tip: Probing magnetoelectric coupling at the nanoscale. Natl Sci Rev, 2019, 6: 626-628

3 Landau L D, Lifshitz E M. Electrodynamics of Continuous Media. Pergamon: Oxford, 1960

4 Dzyaloshinskii I E. On the magneto-electrical effects in antiferromagnets. Sov Phys JETP, 1960, 10: 628-629

5 Astrov D N. The magnetoelectric effect in antiferromagnetics. Sov Phys JETP, 1960, 11: 708-709

6 Rado G T, Folen V J. Observation of the magnetically induced magnetoelectric effect and evidence for antiferromagnetic domains. Phys Rev Lett, 1961, 7: 310-311

7 Nan C W, Bichurin M I, Dong S, et al. Multiferroic magnetoelectric composites: Historical perspective, status, and future directions. J Appl Phys, 2008, 103: 031101

8 Scott J F. Room-temperature multiferroic magnetoelectrics. NPG Asia Mater, 2013, 5: e72

9 Fiebig M. Revival of the magnetoelectric effect. J Phys D-Appl Phys, 2005, 38: R123-R152

10 Wang K F, Liu J M, Ren Z F. Multiferroicity: The coupling between magnetic and polarization orders. Adv Phys, 2009, 58: 321-448

11 Martin L W, Chu Y H, Ramesh R. Advances in the growth and characterization of magnetic, ferroelectric, and multiferroic oxide thin films. Mater Sci Eng-R-Rep, 2010, 68: 89-133

12 Hu J M, Nan C W, Chen L Q. Perspective: Voltage control of magnetization in multiferroic heterostructures. Natl Sci Rev, 2019, 6: 621624

13 Lu C, Wu M, Lin L, et al. Single-phase multiferroics: New materials, phenomena, and physics. Natl Sci Rev, 2019, 6: 653-668

14 Tian G, Yang W, Chen D, et al. Topological domain states and magnetoelectric properties in multiferroic nanostructures. Natl Sci Rev, 2019, 6: 684-702

15 Huyan H, Li L, Addiego C, et al. Structures and electronic properties of domain walls in $\mathrm{BiFeO}_{3}$ thin films. Natl Sci Rev, 2019, 6: 669-683 